\title{
A 22-Year Data Set of Surface LongwaVe FluXes in the Arctic
}

Final Report on DOE/ARM Project 0007366

\author{
P.I.: Dr. Jennifer Francis
}

Institute of Marine and Coastal Sciences, Rutgers University

\section{TOVS-Derived Downwelling Longwave Flux April Mean 1980 to 2001}
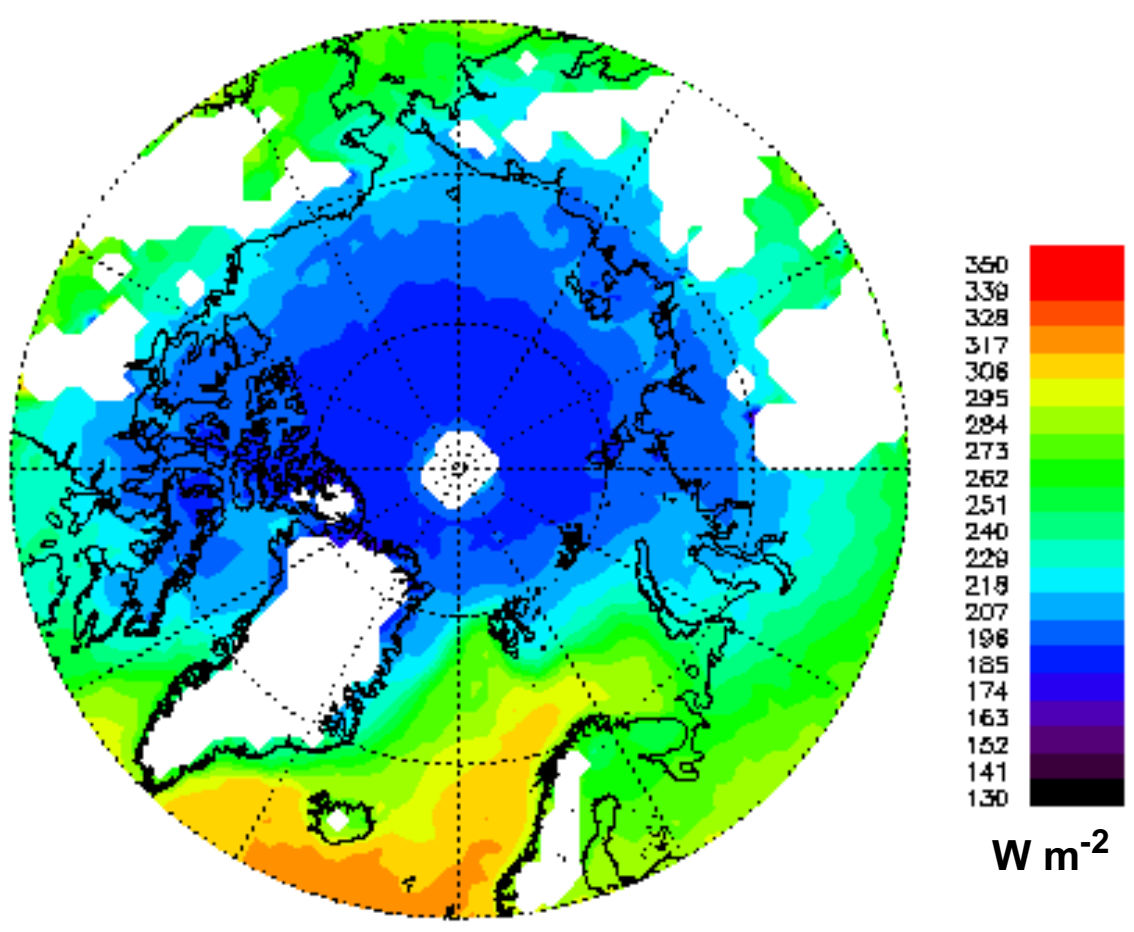


\section{Project Summary}

A reliable estimate of the surface downwelling longwave radiation flux (DLF) is a glaring void in available forcing data sets for models of Arctic sea ice and ocean circulation. We have developed a new method to estimate the DLF from a combination of satellite sounder retrievals and brightness temperatures from the TIROS Operational Vertical Sounder (TOVS), which has flown on NOAA polar-orbiting satellites continuously since late 1979. The overarching goal of this project was to generate a 20-year data set of surface downwelling longwave flux measurements from TOVS data over the Arctic Ocean. Daily gridded fields of DLF were produced with a spatial resolution of $(100 \mathrm{~km})^{2}$ north of $60^{\circ} \mathrm{N}$ for 22.5 years rather than only 20. Surface measurements from the field station at Barrow, AK -- part of the Atmospheric Radiation Measurement (ARM) Program -- and from the Surface Heat Budget of the Arctic (SHEBA) were used to validate the satellite-derived fluxes and develop algorithm improvements for conditions that had resulted in systematic errors in early versions of the algorithm. The resulting data set has already been sent to two other investigators for incorporation into their research, and we will soon complete preparations to send the products to the National Snow and Ice Data Center and ARM data archive, where it can be disseminated to the scientific community.

\section{Accomplishments}

\section{Transition to a neural network}

When the algorithm for deriving DLFs from TOVS retrievals and combinations of brightness temperatures was first developed in the late 1990s [Francis, 1997], the satellite information was ingested by a traditional forward radiative transfer model called Streamer [Key and Schweiger, 1998]. Our original plan was to perform the validation work first using gridded TOVS retrievals in Streamer, but it soon became clear that because of the intense drain on computing resources by the forward RT model, it made sense to convert the algorithm first to a neural network version of the RT model called FluxNet [Key and Schweiger, 1998]. With assistance from the developers of FluxNet, we successfully transferred our method for computing longwave fluxes to the neural network system, which increased the computing speed by an order of magnitude with no perceptible decrease in accuracy. This transition required a great deal of coding, testing, debugging, and intercomparison that had not been originally planned, but the task was successfully completed and the benefits were tremendous. Not only were we able to process (and reprocess) the data more quickly, but we were able to ingest Level-2 (orbital) retrievals rather than gridded values, which increased our temporal resolution and greatly enhanced our ability to identify problems in the algorithm.

\section{Algorithm improvements}

Having a narrow time window for a DLF estimate from Level-2 retrievals rather than a daily average enabled us to visually examine bulk cloud properties near the time of satellite overflight. SHEBA and NSA radar/lidar retrievals were compared to cloud conditions retrieved using the DLF algorithm. Improvements were developed to enhance the algorithm's performance in extremely cold, clear conditions as well as during summer when low-level water clouds dominate. Modifications were made to identify likely fog and low-level ice cloud conditions, and to better differentiate clouds from clear sky in summer. We recognize, however, that perfect agreement is impossible, as satellite retrievals integrate conditions over a $(100-\mathrm{km})^{2}$ area, while SHEBA and NSA measurements are a point in time and space. This incompatibility should result in the 
extremes being damped by the satellite retrievals, which is exactly what we see in comparisons. Retrieved DLFs have been validated with nearly 5 years of flux measurements from the Surface Heat Balance of the Arctic (SHEBA) field program (Fig. 1) and the ARM/NSA site (Fig. 2). During the SHEBA year we find a bias of only $3.2 \mathrm{Wm}^{-2}$ and correlations of 0.86 . During almost 4 years of NSA comparisons the bias is $6.5 \mathrm{~W} \mathrm{~m}^{-2}$ and the RMSEs are about $30 \mathrm{~W} \mathrm{~m}^{-2}$.

\section{0-year DLF product}

The primary objective of this project to generate a 20-year data set of surface downwelling longwave fluxes from TOVS retrievals was accomplished, and then some. In fact, we were able to extend the data record to 22.5 years, as similar TOVS instruments have flown continuously on NOAA polar orbiting satellite platforms from mid-1979 through 2001. Daily products have been completed for the region north of $60^{\circ} \mathrm{N}$ on a $(100 \mathrm{~km})^{2}$ grid. Monthly mean fluxes calculated from the entire record are presented in Fig. 3.

Our DLF product is already being used to validate the NASA/GISS GCM (Fig. 4, bottom) and investigate causes for observed increases in the duration of the Arctic melt season. Packaging of the data set for archival and distribution is nearly completed.

\section{Preliminary analyses}

While this activity was not part of the proposed research, we have begun to analyze spatial and temporal patterns in the 22.5-year record of TOVS-derived DLFs. Seasonal trends in DLF reveal intriguing patterns of change (Fig. 5), but work is needed to ascertain the causes and impacts of these patterns. We see that DLF has apparently increased over most of the Arctic during the period from 1980 to 2001, consistent with observed sea ice decrease, increased ice melt season length, increased surface temperatures, and decreased permafrost. A follow-on proposal to ARM has been submitted to investigate these trends -- identify their causes, and assess their impacts on modeled sea ice behavior.

\section{References}

Chen, Y., J.R. Miller, and J.A. Francis, 2003: Observed and modeled relationships among Arctic climate variables. J. Geophys. Res., 108, 4799, doi:10.1029/2003JD003824.

Francis, J.A. 1997: A method to derive longwave fluxes at the Arctic surface from TIROS operational vertical sounder data, J. Geophys. Res., 102, 1795-1806.

Key, J. and A.J. Schweiger, 1998: Tools for atmospheric radiative transfer: Streamer and FluxNet, Computers and Geosciences, 24(5), 443-451. 


\section{Figures}

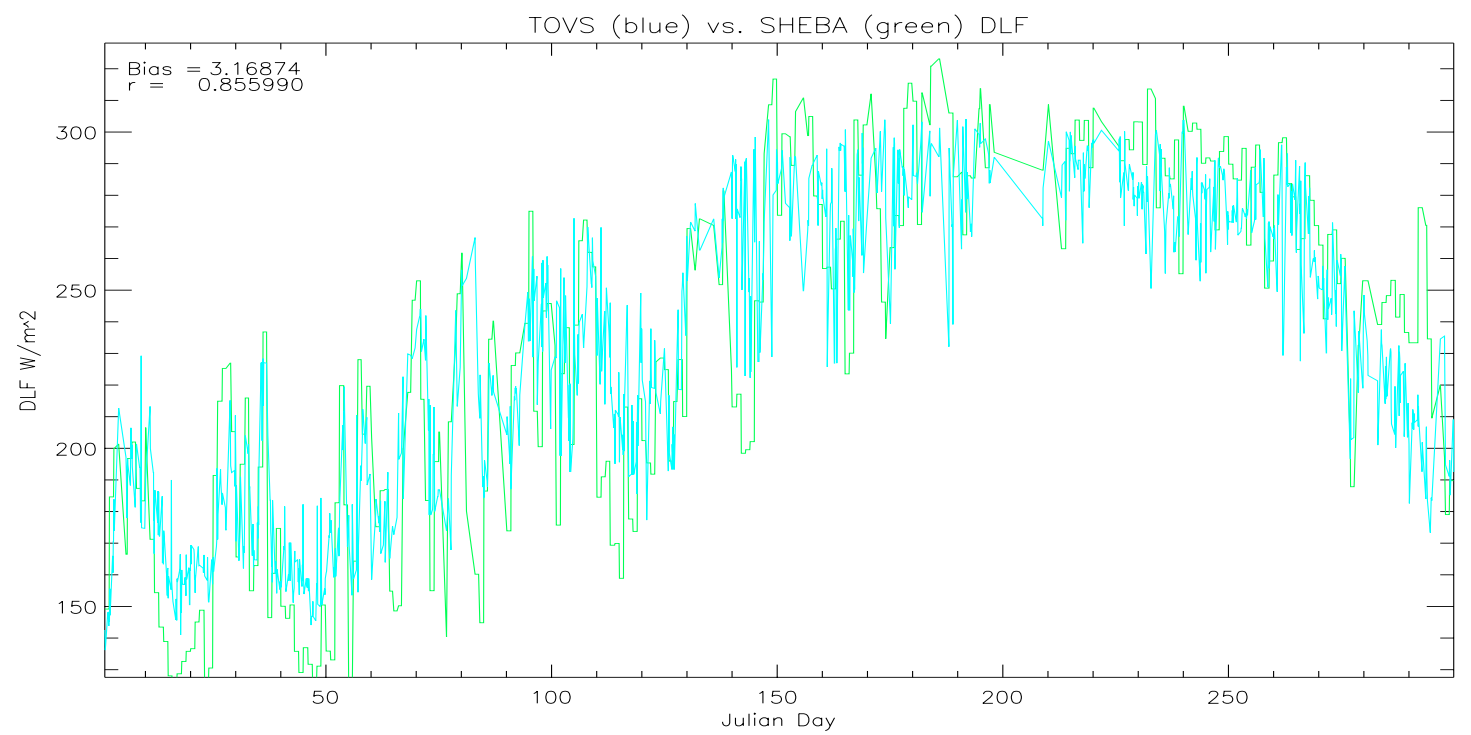

Figure 1: Comparison of daily-average surface downwelling longwave flux calculated from Level-2 (orbital) TOVS retrievals and brightness temperatures (cyan) with daily-averaged measurements from the SHEBA field experiment (green) from 1 January through October 1998. 

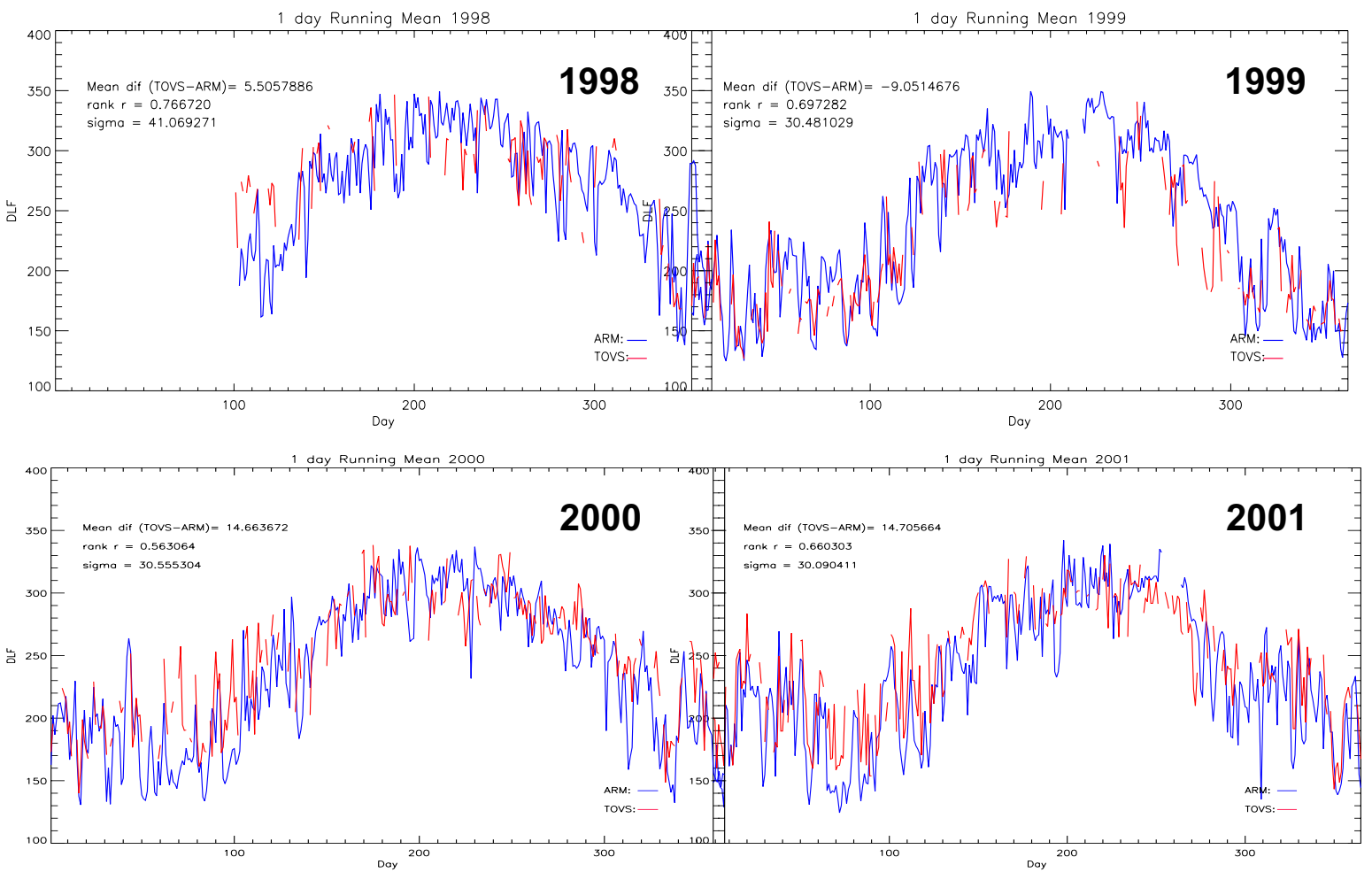

Figure 2: Comparison of daily-mean TOVS-derived DLF(red) with measured DLF from the ARM NSA site (blue) for 1998 through 2001. 

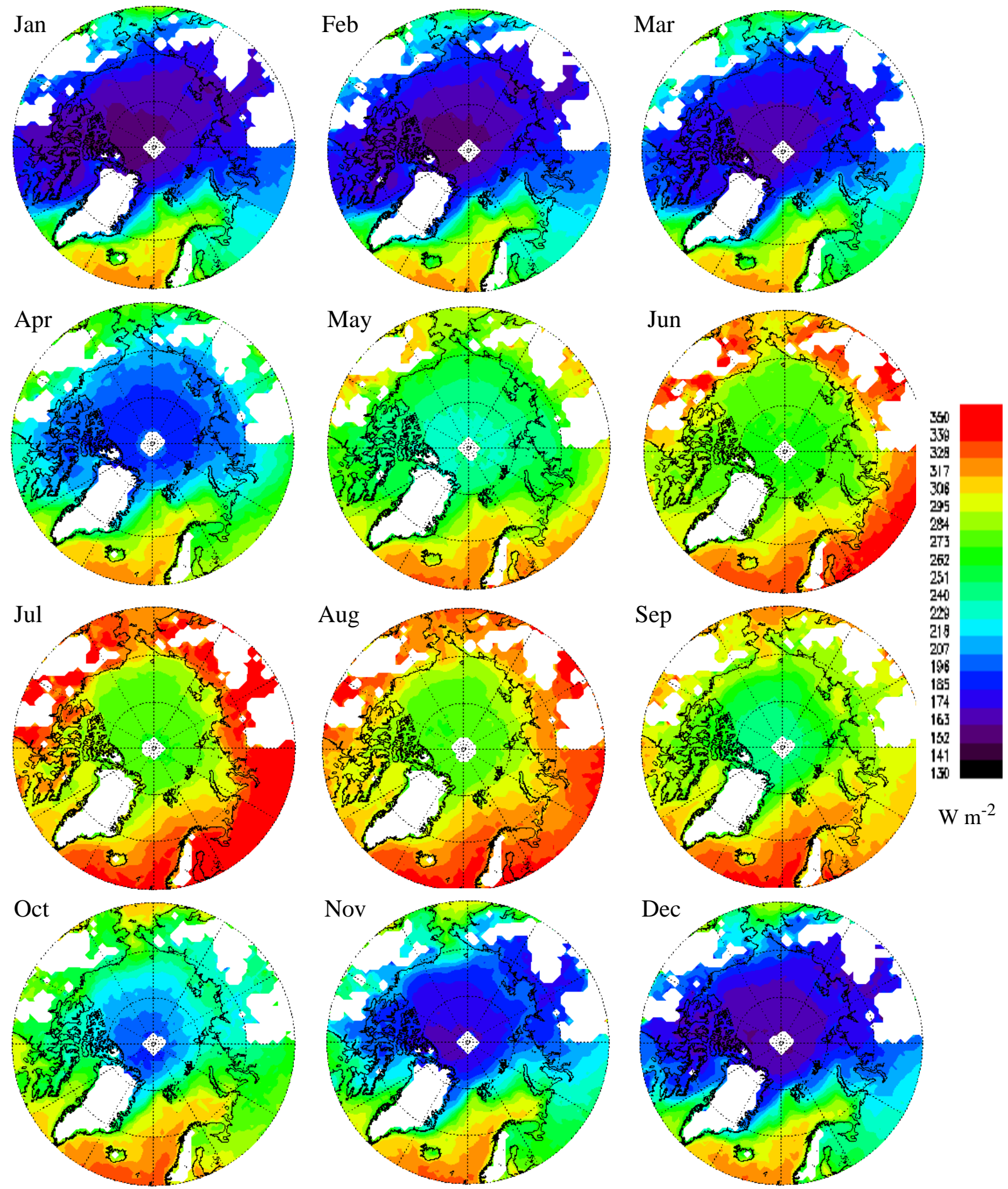

$\mathrm{W} \mathrm{m}^{-2}$

Figure 3: Monthly mean downwelling longwave fluxes derived from TOVS retrievals and radiances from 1979 to 2001 . 

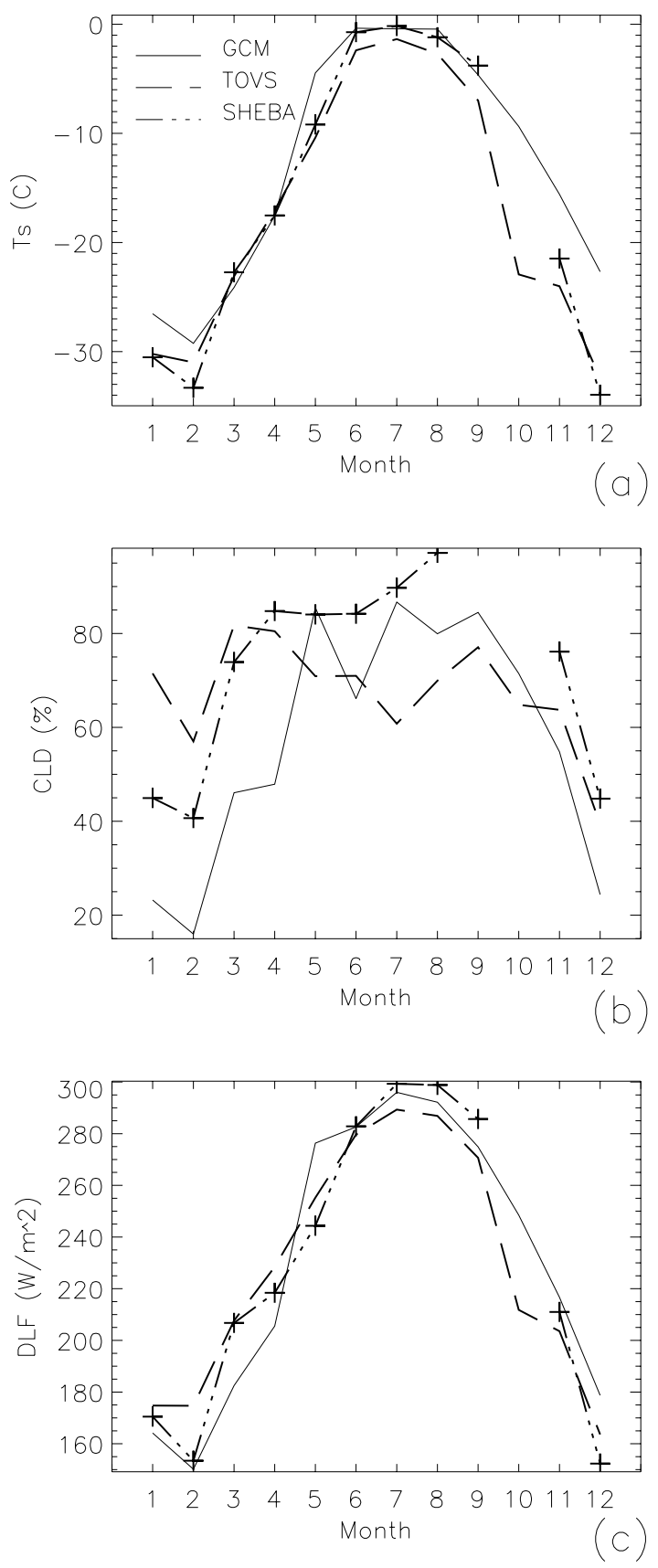

Figure 4: Comparison of annual cycles in surface temperature (a), cloud cover (b) and downwelling longwave flux (c) from the NASA/Goddard Institute for Space Studies (GISS) GCM (solid), TOVS retrievals (dash), and SHEBA measurements (dash-dot). Monthly mean values are plotted from Oct. 1997 to Sept. 1998). From Chen et al. [2003]. 

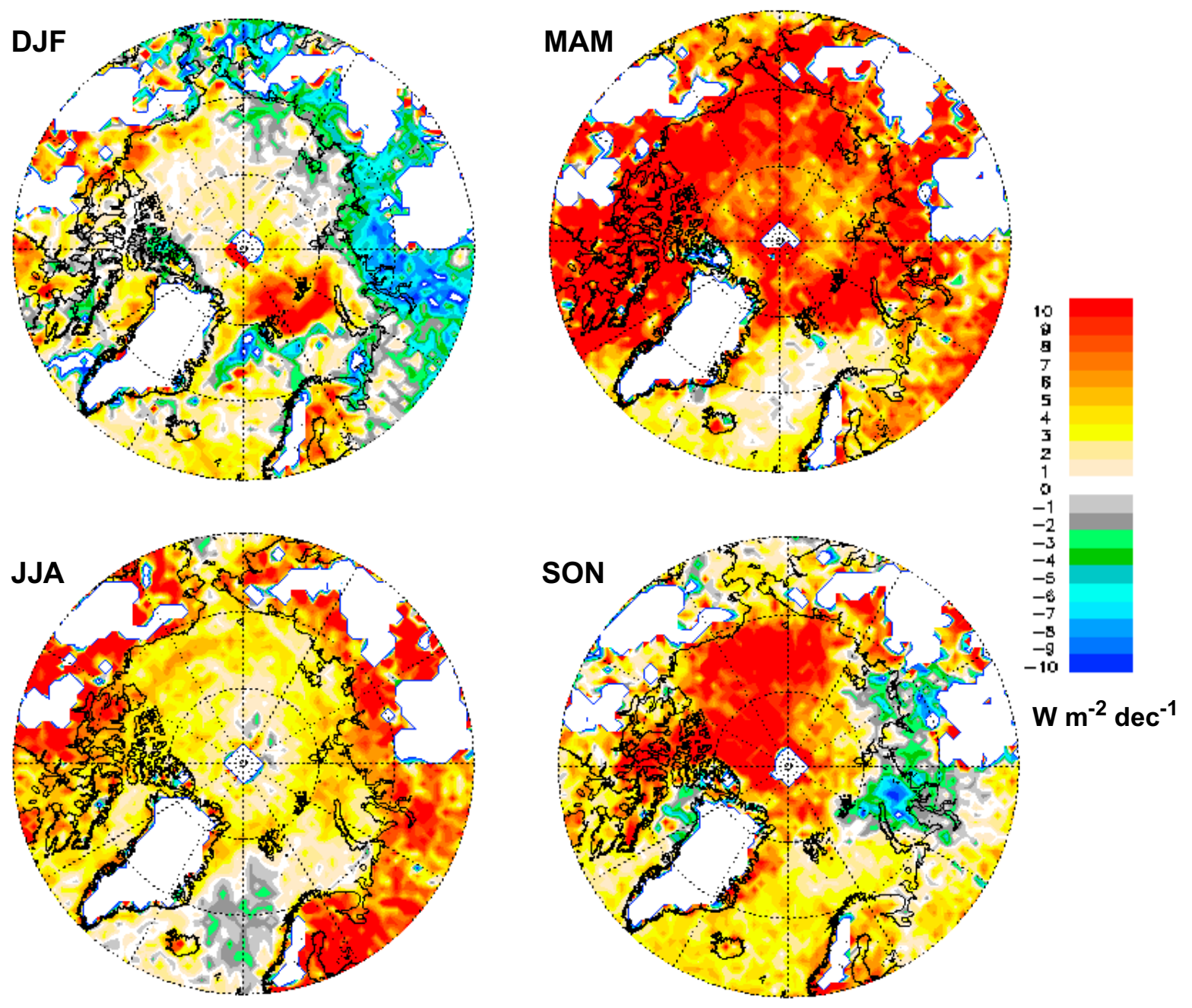

Figure 5: Seasonal decadal trends [W m ${ }^{-2} \mathrm{dec}^{-1}$ ] in TOVS-derived DLF from 1980 to 2001. The upper-left plot is for winter (DJF), upper right is spring (MAM), lower left is summer (JJA), and lower right is autumn (SON). TOVS retrievals are not obtained in areas of high elevation, such as Greenland. 